\title{
High efficacy of gefitinib in the treatment of EGFR mutation-positive advanced non-small cell lung adenocarcinoma: A case report
}

\author{
ZHONGCHAO WANG ${ }^{1,2}$ and JIANJUN CHU ${ }^{2}$ \\ ${ }^{1}$ Department of Radiotherapy, Xinyi People's Hospital, Xuzhou, Jiangsu 221400; ${ }^{2}$ Department of Radiotherapy, \\ The Fourth Affiliated Hospital of Soochow University, Wuxi, Jiangsu 214062, P.R. China
}

Received October 30, 2013; Accepted May 15, 2014

DOI: $10.3892 / \mathrm{ol} .2014 .2269$

\begin{abstract}
Epidermal growth factor receptor (EGFR) tyrosine kinase inhibitors (TKIs), such as gefitinib and erlotinib, are known to play a significant role in EGFR mutation-positive non-small cell lung cancer. When an EGFR mutation is found, gefitinib and erlotinib have been shown to have significant roles in the treatment of untreated advanced NSCLC. This study reports an EGFR mutation in NSCLC treated with gefitinib and is notable due to the patient's marked improvement following a shorter than average duration of treatment with gefitinib. The present study reports the case of a 58-year-old male smoker with a dry cough. Computed tomography revealed a mass in the left inferior lobe of the lung. The patient was subsequently diagnosed with advanced lung adenocarcinoma, and an EGFR mutation (in-frame deletions of E746-A750 in exon 19) was found. The patient received multiple rounds of chemotherapy, followed by gefitinib maintenance therapy for 3 months. Later on, a grade 1 acne-like rash developed on the face and back that lasted throughout the treatment. Currently, the patient is stable, with no evidence of disease progression. The present study describes the disease and the treatment using gefitinib.
\end{abstract}

\section{Introduction}

Non-small cell lung cancer (NSCLC) is a common cause of cancer-related mortality in China. Although several novel targeted anticancer agents are available, platinum-based chemotherapy remains the first-line therapy, achieving better progression free survival (PFS) rates than non-platinum-based regimens (1).

Correspondence to: Mr. Jianjun Chu, Department of Radiotherapy, The Fourth Affiliated Hospital of Soochow University, 200 Huihe Road, Wuxi, Jiangsu 214062, P.R. China

E-mail:wschujianjun@163.com

Key words: gefitinib, epidermal growth factor receptor, tyrosine kinase inhibitors, non-small cell lung cancer
Epidermal growth factor receptor (EGFR) tyrosine kinase inhibitors (TKIs), such as gefitinib and erlotinib, have been shown to play a significant role in the treatment of untreated advanced NSCLC, particularly in NSCLC patients with EGFR mutations. Two phase III studies (NEJ002 and WJTOG3405) showed an improved PFS rate in NSCLC patients harboring sensitizing EGFR mutations $(2,3)$. Therefore, gefitinib and erlotinib can be used as the first-line treatment of patients with advanced or metastatic NSCLC with activating EGFR mutations.

The present study describes the case of a patient with an EGFR mutation in NSCLC treated with gefitinib, achieving a marked efficacy. Patient provided written informed consent.

\section{Case report}

A 58-year-old male, with no significant medical history developed a dry cough in November 2011. The patient had previously smoked 10 cigarettes per day for 30 years, but stopped smoking in January 2012. A chest computed tomography (CT) scan revealed a mass in the left inferior lobe, resulting in the patient being admitted to The Fourth Affiliated Hospital of Soochow University (Wuxi, Jiangsu, China). A brain CT scan showed no evidence of any distant metastasis. A bone emission CT (ECT) scan showed multiple bone metastases. Positron emission tomography-CT scan showed a large soft-tissue mass in the left inferior lobe of the lung, and multiple masses in the right lung, right adrenal glands and bones. Fiber bronchoscopy found cancer cells in the section analyzed, and histopathology revealed an adenocarcinoma. The patient was diagnosed with adenocarcinoma by a CT-guided percutaneous core needle biopsy. The clinical stage was stage IV. EGFR mutations were detected using the peptide nucleic acid-locked nucleic acid polymerase chain reaction clamp method. An EGFR mutation was found with deletions in E746-A750 of exon 19.

Due to a metastasis in the eleventh and twelfth thoracic vertebrae that caused spinal cord compression, the patient initially received $30 \mathrm{~Gy}$ radiation of $3 \mathrm{~Gy}$ per fraction. Following this, $1.6 \mathrm{~g}$ gemcitabine (GEM $1.0 \mathrm{~g} / \mathrm{m}^{2}$ ) was administered on days one and eight, and $30 \mathrm{mg}$ cisplatin (DDP) was administered on days one to four. Six cycles were administered every three weeks (February-July, 2012). No adverse events (AEs) were reported. 

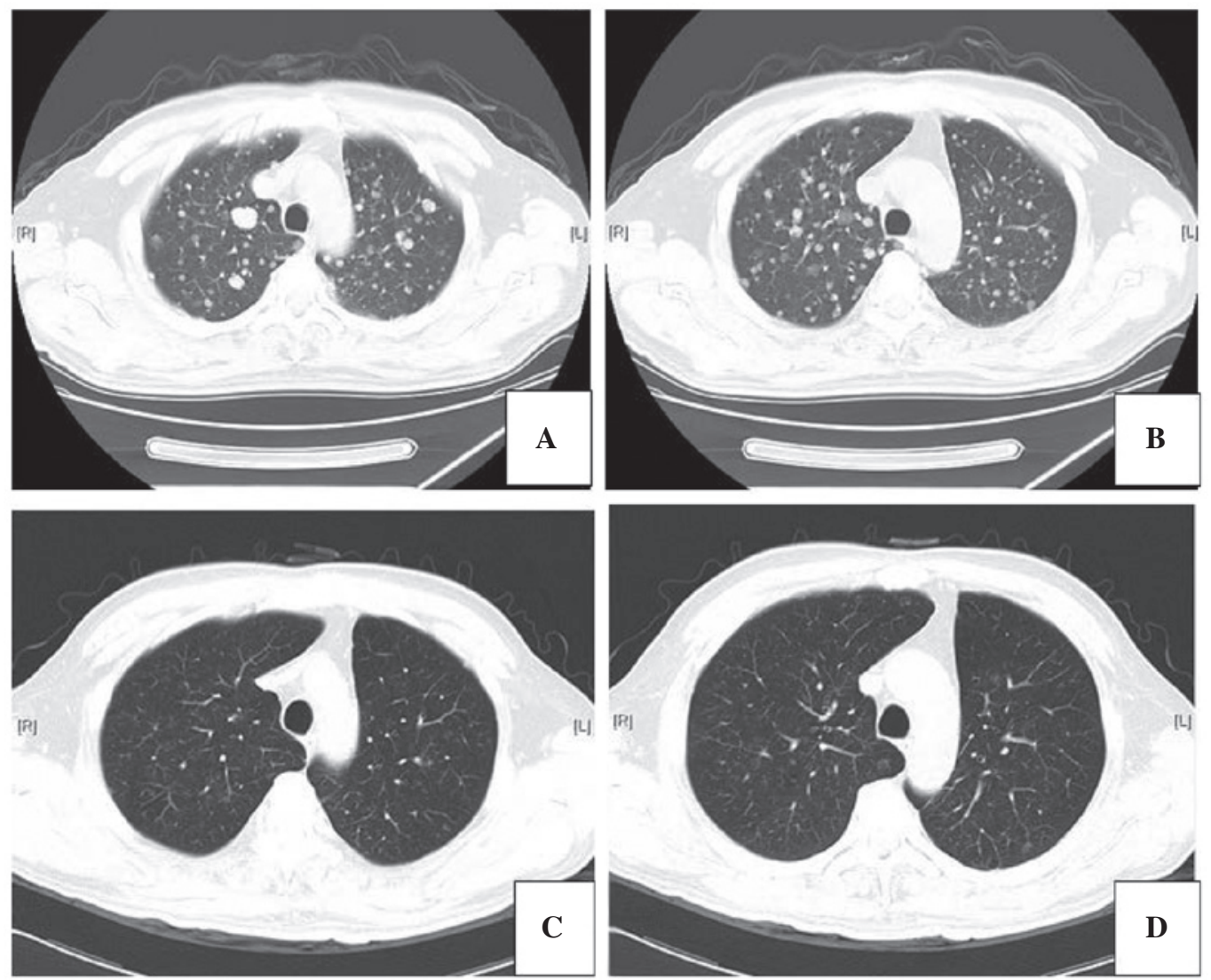

Figure 1. Axial chest computed tomography (CT) scans. (A and B) Scans prior to treatment with gefitinib. (C and D). Scans two months after treatment with gefitinib.

A chest CT scan carried out in August 2012 showed residual disease in the left inferior lobe of the lung $(2 \mathrm{~cm}$ in diameter), and metastatic lesions of the right lung, the right adrenal glands and bone were stable. Chemotherapy was subsequently continued. The patient received four cycles of chemotherapy consisting of $1.6 \mathrm{~g}$ GEM on days one an eight, and $30 \mathrm{mg}$ DDP on days one to four. The last chemotherapy treatment was on November 24, 2012. No AEs were reported. A CT scan of the thorax was assessed as stable. In May 2013, another ECT scan revealed one new lesion in the right femur, indicating progression of the disease. The patient received another 30 Gy radiation, $3 \mathrm{~Gy} / \mathrm{FX}$.

Another Chest CT scan showed widespread metastases in the right and left lung (Fig. 1). The patient was consequently administered $250 \mathrm{mg}$ oral gefitinib once daily in June 2013. A grade 1 acne-like rash developed on the face and back, which was treated with $4.5 \mathrm{~g}$ piperacillin-tazobactam twice daily for 5 days. The rash lasted the clinical course of the treatment. Chest CT scans showed that the metastatic tumors were improved following gefitinib treatment (Fig. 1). To date, the disease remains stable and the patient continues to receive gefitinib orally.

\section{Discussion}

The current study presents the case of an NSCLC patient with an EGFR mutation treated with gefitinib. For an unknown EGFR status, platinum-based chemotherapy remains in use as the first-line management of NSCLC $(4,5)$. However, gefitinib is the first targeted agent to be approved for the treatment of EGFR mutation-positive lung adenocarcinoma, which has showed evident clinical efficacy, particularly among non-smokers, East Asian females and patients with adenocarcinoma (6,7). EGFR mutation analyses have demonstrated that patients with activating EGFR gene mutations obtained more benefit than those of non-EGFR gene mutations (8). A recent meta-analysis also showed that gefitinib yielded a statistically significant benefit in progression-free survival compared with gefitinib-free therapy (9).

Drug-related side-effects are significantly more common in patients receiving gefitinib therapy (9). EGFR-TKI therapy causes the development of a rash in numerous patients (10). In the patient of the present study, a grade 1 acne-like rash developed on the face and back following the start of gefitinib treatment. The rash persisted throughout the treatment period. Rash development has been associated with EGFR-TKI efficacy in NSCLC, and a meta-analysis previously showed that a skin rash was an independent predictive factor for progression and survival in EGFR TKI-treated NSCLC patients (10). Other adverse events, such as diarrhea, dry skin, pruritus and paronychia, have also been notable in the current literature . Therefore, the status of the patient should be considered prior to gefitinib treatment to ensure that the best therapy is being selected.

EGFR-TKI therapy may prolong life expectancy. Despite the fact that the treatment time is short, we believe that if used long-term, maintenance therapy would benefit those patients with EGFR mutations. Furthermore, a retrospective 
study analyzed 301 long-term NSCLC survivors to confirm which patient may benefit most from erlotinib treatment (11). The long-term gefitinib benefits were not only relative to good prognostic factors (e.g., good performance status, never-smoker status and the female gender), but also included negative factors. A study of 124 advanced NSCLC patients treated with chemotherapy [64 $(51.6 \%)$ patients treated with gefitinib] as the initial therapy revealed that $8 \%$ of the patients survived for $>5$ years (12). In addition, another study reported that three refractory NSCLC patients survived for $>3$ years with gefitinib treatment (13). It was concluded that patients with EGFR mutations gained the greater benefit, particularly those who were older or had a poor performance status, from first-line EGFR-TKI therapy (12).

In conclusion, this study suggests that gefitinib therapy has a significant role in advanced lung adenocarcinoma. A limitation of the current study was the small sample size. Future studies with larger sample size are required to investigate EGFR mutations.

\section{References}

1. $\mathrm{Yu} Y, \mathrm{Xu} X, \mathrm{Du} \mathrm{Z}$ and Shi M: Non-platinum regimens of gemcitabine plus docetaxel versus platinum-based regimens in first-line treatment of advanced non-small cell lung cancer: a meta-analysis on 9 randomized controlled trials. Cancer Chemother Pharmacol 69: 1265-1275, 2012.

2. Mitsudomi T, Morita S, Yatabe Y, Negoro S, Okamoto I, Tsurutani $\mathrm{J}$, et al; West Japan Oncology Group: Gefitinib versus cisplatin plus docetaxel in patients with non-small-cell lung cancer harbouring mutations of the epidermal growth factor receptor (WJTOG3405) an open label, randomised phase 3 trial. Lancet Oncol 11: 121-128, 2010.

3. Maemondo M, Inoue A, Kobayashi K, Sugawara S, Oizumi S, Isobe $\mathrm{H}$, et al; North-East Japan Study Group: Gefitinib or chemotherapy for non-small-cell lung cancer with mutated EGFR. N Eng J Med 362: 2380-2388, 2010.
4. Mok TS, Wu YL, Thongprasert S, Yang CH, Chu DT, Saijo N, et al: Gefitinib or carboplatin-paclitaxel in pulmonary adenocarcinoma. N Eng J Med 361: 947-957, 2009.

5. Gridelli C, Butts C, Ciardiello F, Feld R, Gallo C and Perrone F: An international, multicenter, randomized phase III study of first-line erlotinib followed by second-line cisplatin/gemcitabine versus first-line cisplatin/gemcitabine followed by second-line erlotinib in advanced non-small-cell lung cancer: treatment rationale and protocol dynamics of the TORCH trial. Clin Lung Cancer 9: 235-238, 2008.

6. Thatcher N, Chang A, Parikh P, Rodrigues Pereira J, Ciuleanu T, von Pawel J, et al: Gefitinib plus best supportive care in previously treated patients with refractory advanced non-small-cell lung cancer: results from a randomised, placebo-controlled, multicentre study (Iressa Survival Evaluation in Lung Cancer). Lancet 366: $1527-1537,2005$

7. Maruyama R, Nishiwaki Y, Tamura T, Yamamoto N, Tsuboi M, Nakagawa K, et al: Phase III study, V-15-32, of gefitinib versus docetaxel in previously treated Japanese patients with non-small-cell lung cancer. J Clin Oncol 26: 4244-4252, 2008.

8. Ebi N, Semba H, Tokunaga SJ, Takayama K, Wataya H, Kuraki T, et al; Lung Oncology Group in Kyushu, Japan: A phase II trial of gefitinib monotherapy in chemotherapy naïve patients of 75 years or older with advanced non-small cell lung cancer. J Thorac Oncol 3: 1166-1171, 2008.

9. Zhou H, Zeng C, Wang LY, Xie H, Zhou J, Diao P, et al: Chemotherapy with or without gefitinib in patients with advanced non-small-cell lung cancer: a meta-analysis of 6,844 patients. Chin Med J (Engl) 126: 3348-3355, 2013.

10. Petrelli F, Borgonovo K, Cabiddu M, Lonati V and Barni S: Relationship between skin rash and outcome in non-small-cell lung cancer patients treated with anti-EGFR tyrosine kinase inhibitors: a literature-based meta-analysis of 24 trials. Lung Cancer 78: 8-15, 2012.

11. Schumann C, Heigener D, Dittrich I, et al: Long term benefit from erlotinib treatment is independent of prognostic factors and therapeutic response (abstract 9146). Eur J Cancer 7: 1, 2009.

12. Kaira K, Takahashi T, Murakami H, Tsuya A, Nakamura Y, Naito T, et al: Long-term survivors of more than 5 years in advanced non-small cell lung cancer. Lung Cancer 67: 120-123, 2010.

13. Nakatomi K, Soda H, Kitazaki T, Nakano H, Uchida K, Urabe S, et al: Long-term survival in three patients with metastatic non-small cell lung cancer treated with gefitinib. Lung Cancer 52: 253-255, 2006. 